\title{
CAPACITANCE-VOLTAGE DIFFERINTEGRAL CHARACTERISTICS OF FRACTAL FIELD-EFFECT DEVICE
}

\author{
V.M. Onufrienko \\ National University “Zaporizhzhia Polytechnic”, Zaporizhzhia, Ukraine \\ e-mail: onvm@i.ua
}

In the present paper, we use the theoretical model of fractal elements to study the charge and capacitance-voltage differintegral characteristics of a fractal field-effect device. The control of the charge channel and the capacity of a fractal nanotransistor is revealed by changing the scaling of the nanolayer, the charge density in which is determined from the sum of the charges of electrons, holes, and ionized impurities. The significant influence of fractal charge distribution carriers on the device capacitance at high frequencies is noted. This fact theoretically confirms the possibility of increasing the capacitance of metaloxide-semiconductor structures to increase the efficiency of the field-effect-transistors. The comparison of the obtained expressions shows the strong influence of fractal medium on the electric field size and charge density in a semiconductor that is followed by the change of capacitive device parameters. The differentialintegral method for modeling artificial metamaterial fractal radioelements with necessary electrical characteristics is proposed.

Keywords: field-effect nanotransistor, fractal nanolayer, differintegral characteristics, potential, charge density, capacitance; capacitance-voltage characteristic modeling..

Received 22.09.2020; Received in revised form 20.11.2020; Accepted 02.12.2020

\section{Introduction}

The study of metamaterials in the frequency bands where the effects occurring in devices with a semiconductor medium and distributed negative capacities and inductances [1] are important is one of promising trends in the development of modern electronics. Researchers from Purdue University have suggested the technological implementation of a ferroelectric metamaterial layer with a negative capacitance, which increases the transistor efficiency [2].

By optimizing material parameters and tuning negative capacitance, negativecapacitance field-effect transistors (FET) can achieve 2.5 times higher energy efficiency than conventional metal-oxide-semiconductor (MOS) [3]. The FET channel is described by the Poisson-Schrödinger equation to calculate electrostatics and quantum mechanical states.

We consider the possibility of charge and capacity control in radioelectronic devices based on MOS-FET that operate on the basis of changing the conductivity of a channel between a source (S) and a drain (D) in a fractally configured semiconductor region near the boundary with a dielectric layer under the electric field action.

The interest to the development of such elements in the integrated circuit is explained by the fact that most modern analog and digital integrated circuits are executed by MOStechnology [4]. The theory of a fractal layer [5,6] at the interface between two media is based on the determination of the fractional differential elements.

The definition of a compact covering of the heterogeneous set with introduction of dilatations $l_{i(k)}$ ( $k$ is the number of coat generations, $i$ is the number of subsets in the $k$-th generation) with their diminution in the following generations of a coat $l_{i(k+1)}<l_{i(k)}$ (Fig. 1), gives a functional connection of number of polygonal vertexes $N_{l_{i(k+1)} l_{i(k)}}=f\left(l_{i(k)} / l_{i(k+1)}\right)$. The projections of the cover links onto $O X$ axis satisfy the functional equation $f\left(\Delta x_{i(k)} / \Delta x_{i(k+1)}\right) \cdot f\left(\frac{\Delta x_{i(k)} / \Delta x_{i(k+2)}}{\Delta x_{i(k)} / \Delta x_{i(k+1)}}\right)=f\left(\Delta x_{i(k)} / \Delta x_{i(k+2)}\right), \quad$ a smooth solution of which exists and is unique in the form of a power function (see, for example, [7]): 


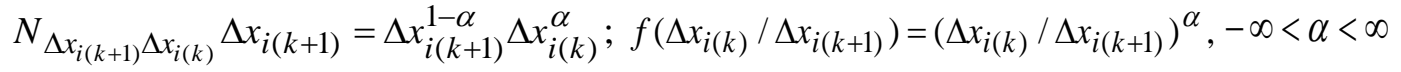

The sum $\quad \gamma(\alpha)\left(\sum_{i=1}^{\infty} l_{i}\right)^{\alpha} \quad$ approximates all the considered contour $L_{i(k)}=\sum l_{i(k)} ; \quad L_{i}=\left(L_{i(k)}\right)^{\alpha}=\left(\sum l_{i(k)}\right)^{\alpha}$.

The passage to the limit gives a fractional differintegral $d^{\alpha(x)} x$ with variable exponent $\alpha(x)$ in formulas, so a fractional exterior derivative can be defined by the equation $d^{\alpha(x)} L(x)={ }_{a} D_{x}^{\alpha(x)} L(x) d^{\alpha(x)} x$ where

$$
d^{\alpha} x=\frac{x_{i}^{\alpha(x)-1}}{\Gamma(\alpha(x))} d x
$$

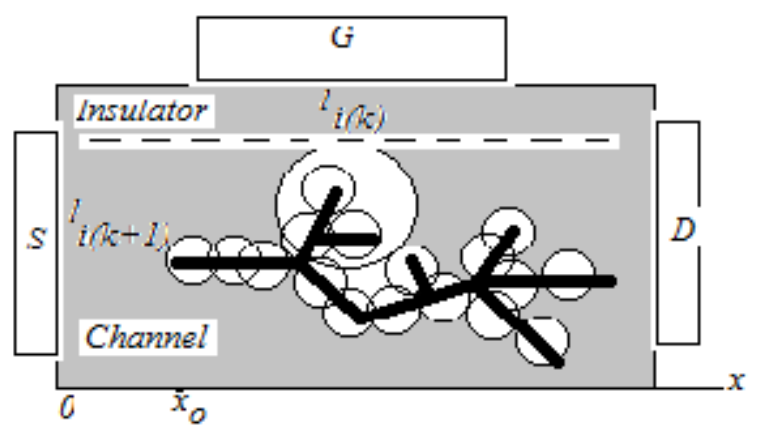

Fig. 1. Geometric fractal element coat $l_{i(k+1)}<l_{i(k)}$ of metal-oxide-semiconductor channel.

The fractional derivative $\left({ }_{a} D_{x}^{\alpha(x)} L\right)(x)$ is used in the Riemann-Liouville form with a variable scaling index (see, for example, [8])

$$
\left({ }_{a} D_{x}^{\alpha(x)} f\right)(x)=\frac{1}{\Gamma(\alpha(x))} \int_{a}^{x} \frac{f(t)}{(x-t)^{1-\alpha(x)}} d t .
$$

\section{Charges on fractal elements}

The fractal element of a charge distribution $\rho^{(\alpha)}(x) d x=\rho(x) d^{\alpha} x$ in volume of oxide MOS-structure ( $x$ here corresponds to the demarcation metal-ferroelectric, the ferroelectric-semiconductor border coordinate $x=x_{0}$ (Fig.1)) induces charges: $d^{\alpha} Q_{g}$ in metal and $d^{\alpha} Q_{s}$ in semiconductor. The field change $d E_{1}$ to the left and $d E_{2}$ to the right from the point $x$, which is caused by these charges, we write down in the form of the equation $x d E_{1}+\left(x_{0}-x\right) d E_{2}+d^{(\alpha)} \varphi_{s}=0$. From here, taking into account the determination of the superficial potential $\varphi_{s}=f\left(Q_{s}\right), d^{\alpha} \varphi_{s}=f^{(\alpha)}\left(Q_{s}\right) d^{\alpha} Q_{s}$, the Gauss theorem and the neutrality condition $d^{\alpha} Q_{g}+d^{\alpha} Q_{s}+\rho^{(\alpha)} d x=0$, for the 
definition of charge in the semiconductor we obtain the equation $-d^{\alpha} Q_{s}\left[x_{0}-\varepsilon_{0 x} f^{(\alpha)}\left(Q_{s}\right)\right]=x \rho(x) d^{\alpha} x$.

After the integration we have expressions for additional charges $Q_{s}^{\prime}$ and $Q_{g}^{\prime}$ induced in the semiconductor and in the metal by the total charge in the ferroelectric material:

$$
\begin{gathered}
Q_{s}^{\prime}=-\int_{0}^{x_{0}} \frac{x}{x_{0}} \rho(x) d^{\alpha} x+\Delta Q ; \quad \Delta Q=\frac{\varepsilon_{0 x}}{x_{0}} \int_{Q_{s}}^{Q_{s}+Q_{s}^{\prime}} f^{(\alpha)}\left(Q_{s}\right) d Q_{s} ; \\
Q_{g}^{\prime}=-\int_{0}^{x_{0}}\left(1-\frac{x}{x_{0}}\right) \rho(x) d^{\alpha} x-\Delta Q .
\end{gathered}
$$

For a fractal element of charge $\rho^{(\alpha)}(x)=Q_{s s}^{\prime} \delta^{(\alpha)}\left(x-x_{0}\right)+Q_{g s}^{\prime} \delta^{(\alpha)}$, which is concentrated on a ferroelectric material surface, we determine sizes of the equivalent surface-bound charges $Q_{s s}^{\prime}, \quad Q_{g s}^{\prime}$. Thus, selection in the model of fractal elements leads to the formation of a fractal configured charge $Q_{s s}=Q_{s}^{\prime}+Q_{s s}^{\prime}$ on the ferroelectricsemiconductor border.

\section{Charge density on fractal configured border}

The construction of a fractal model for the nanolayer capacitance simulation at the metal-semiconductor interface in space as a function of the set is feasible by introducing the differential alpha-form of sets of physical charges (current elements) in ferroelectric and semiconductor metamaterial.

As well as in the classical statement, we determine charge density in a semiconductor by the sum of electron charges $(n)$, holes $(p)$ and ionized impurities $(N)$ [4]. For the nondegenerate semiconductor $\quad \rho(x)=q(-n+p+N), \quad n=n_{i} \exp \left[-\beta\left(\varphi_{F}-\varphi\right)\right]$, $p=n_{i} \exp \left[\beta\left(\varphi_{F}-\varphi\right)\right]$ where $\beta=q /(k T), \quad n_{i}$ is carrier concentration in the intrinsic semiconductor $N=-2 n_{i} \operatorname{sh}\left(\beta \phi_{F}\right), \varphi_{F}$ - potential, are counted from a Fermi level in own exemplar.

As a result, charge density depends on coordinate $x$ in fractal medium as $\rho(x)=2 q n_{i}\left[\operatorname{sh} \beta\left(\phi_{F}-\phi\right)-\operatorname{sh} \beta \phi_{F}\right]$ and satisfies the Poisson's equation

$$
\frac{d}{d x} \frac{d^{\alpha} u}{d x^{\alpha}}=-\frac{\rho(x)}{s_{g}}=\frac{1}{L_{D}^{2}}\left[\operatorname{sh} u_{F}-\operatorname{sh}\left(u_{F}-u\right)\right],
$$

$L_{D}=\sqrt{\varepsilon_{s} /\left(2 \beta q n_{i}\right)}$ is Debye length of screening, $\varepsilon_{s}$ is semiconductor inductivity, $u_{F}=\beta \varphi_{F}, u=\beta \varphi$.

After the integration of the Poisson's equation (4) we obtain $\alpha$ - potential characteristics

$$
\begin{gathered}
D_{x}^{\alpha} u= \pm \frac{\varepsilon_{s}}{\beta} \frac{Q_{1}\left(u_{s}, u_{F}\right)}{L_{D}} ; \\
Q_{1}\left(u_{s}, u_{F}\right)=\frac{\sqrt{2}}{L_{D}} \sqrt{u_{s} \operatorname{sh} u_{F}+\operatorname{ch}\left(u_{F}-u_{S}\right)-\operatorname{ch} u_{F}}
\end{gathered}
$$


The electric field value on the surfaces of the semiconductor $E_{s}^{(\alpha)}=-\left.\frac{1}{\beta}\left(D_{x}^{\alpha} u\right)\right|_{s}$ and the total charge on a semiconductor surface unit are calculated taking into account the obtained equations $E_{s}^{(\alpha)}=-\left.\frac{1}{\beta}\left(D_{x}^{\alpha} u\right)\right|_{s} ; Q_{s}^{(\alpha)}=-\varepsilon_{s} E_{s}^{(\alpha)} \quad$ (according to the Gauss theorem).

We determine the charge density on fractal configured border by integrating (4) from 0 to $x$

$$
\pm \int_{u_{s}}^{u} \frac{d u}{Q_{1}\left(u, u_{F}\right)}=\frac{x^{\alpha}}{\Gamma(1+\alpha)}
$$

\section{Capacity of the fractal conduit channel}

The volume charge of the semiconductor $Q_{s}^{(\alpha)}$, which size depends on a fractalization degree, and bias voltage $V_{G}$ on a metallic electrode results in the dependence of aggregate capacitance per unit area of MOS-structures from $V_{G}$ as $C_{t}^{(\alpha)}=d Q_{G} / d V_{G}$ where a gate specific charge $Q_{G}=-Q_{s}^{(\alpha)}-Q_{s s}$. This is a basis fact of a method for studying MOS-structures and further it is applied by us for determining the fractal structure capacity.

For rather low frequency of a small variable signal enclosed to the gate, the measured capacitance practically coincides with the capacity of the fractal structured oxide

$$
C_{t}^{(\alpha)} \approx C_{0}=\frac{\varepsilon_{0 x}^{(\alpha)}}{x_{0}}, \quad \varepsilon_{0 x}^{(\alpha)}=\frac{\varepsilon_{0 x}}{\frac{1}{\Gamma(1+\alpha)} x_{0}^{\alpha}}
$$

( $\varepsilon_{0 x}$ is the oxide layer dielectric constant, $x_{0}$ is the layer thickness).

For a low-frequency signal and increase in bias voltage the aggregate capacitance is modelled sequentially by the switched-on capacitors $C_{0}$ and $C_{s}^{(\alpha)}$ (capacity of the superficial states $\left.C_{s s}=-d Q_{s s} / d \varphi_{s}=0\right)$ :

$$
\frac{C_{t}^{(\alpha)}}{C_{0}}=\left(1+\frac{C_{0}}{C_{s}^{(\alpha)}}\right)^{-1} ; C_{s}^{(\alpha)}=-\beta \frac{d Q_{s}^{(\alpha)}}{d u_{s}}=\frac{\left.s_{s} q\right)}{Q_{s}^{(\alpha)}}\left[N+2 n_{i} \operatorname{sh}\left(u_{F}-u_{s}\right)\right] .
$$

At high frequencies, when the magnitude of the semiconductor capacitance $C_{s}^{(\alpha)}=-\frac{d Q_{s}^{(\alpha)}}{d \varphi_{s}}$ in the inversion mode is determined by the thickness $x_{d}$ of the depleted region, the fractal structure of the charge carrier distribution is manifested, and the expressions for the capacitance have the form

$$
\frac{C_{t}^{(\alpha)}}{C_{0}}=\left(1+\frac{\varepsilon_{0 x} x_{d}^{(\alpha)}}{\varepsilon_{s} x_{0}}\right)^{-1}
$$




\section{5. $C$ - $V$ characteristics determination and calculation}

To study the rapid switching of the system to inversion mode when the charge of the inversion layer does not have time to be formed that is accompanied by the occurrence of transients, taking into account $\varphi_{s}^{(\alpha)}=\frac{Q_{s}^{(\alpha)}}{C_{0}}+V_{G}^{\prime}$ and integrating the Poisson's equation, we have [9]

$$
\begin{gathered}
\varphi_{s}^{(\alpha)}=-\frac{q}{\varepsilon_{s}} \int_{0}^{x_{d}} x N d^{\alpha} x=-\frac{q N}{\varepsilon_{s}} \frac{x_{d}^{1+\alpha}}{\Gamma(\alpha+2)}, \quad 0<\alpha<1 ; \\
\frac{C_{t}^{(\alpha)}}{C_{0}}=\left(1+\frac{\left(-1 \pm \sqrt{\left.1-a_{d} \frac{2 C_{0}^{2} V_{G}^{\prime}}{q N \varepsilon_{s}}\right)}\right.}{a_{d}}\right)^{-1} ; a_{d}=\frac{2}{\Gamma(\gamma+1)} x_{d}^{\gamma-2}, \quad 1<\gamma \leq 2 .
\end{gathered}
$$

For the scaling indices $\alpha=1, \gamma=2$, the obtained dependences $(8-11)$ coincide with known classical results [4].

We note the significant influence of the fractal distribution of charge carriers on the capacitance, which theoretically confirms the possibility of increasing the capacitance of MOS structures, in particular, to increase the efficiency of the FET.

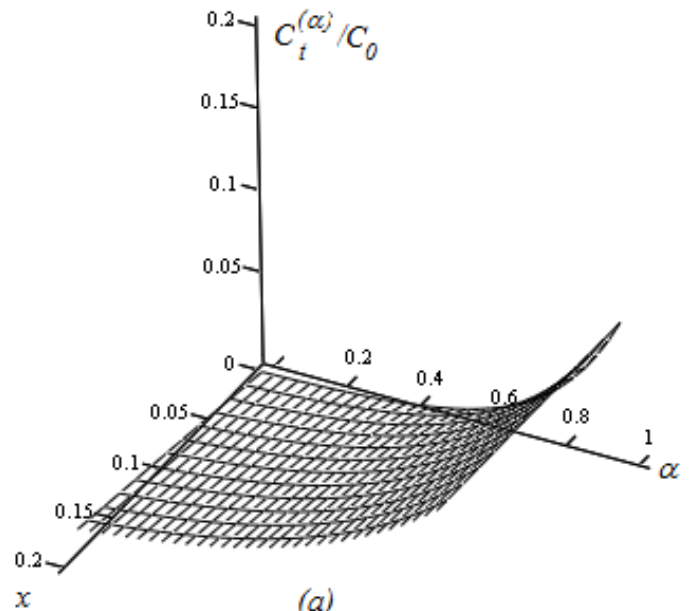

(a)

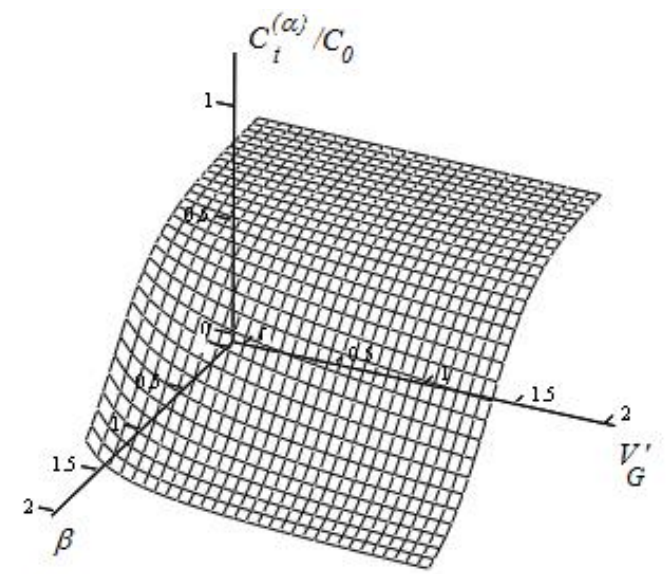

(b)

Fig. 2. Relative capacitance of a fractal silicon MOS structure at high frequencies

depending on layer thickness $x$, fractal scaling $\alpha$ and $\beta$, and voltage $V_{G}^{\prime}$.

Figure 2 shows the results of calculating the ratio $C_{t}^{(\alpha)} / C_{0}$ of the semiconductor capacitance: we plot the graph $(a)$ according to equations (10) depending on the scaling parameter (from $\alpha=0$ to $\alpha=1$ ) and relative thickness $x_{d} / x_{0}$; the graph $(b)$ shows the $C$ - $V$ differintegral characteristics determined by equation (11) depending on the potential $V_{G}^{\prime}$ and the scaling parameter (from $\beta=1$ to $\beta=2$ ). For comparison with the known theoretical and experimental 
results, we used the phenomenological data $|N|=10^{15} \mathrm{~cm}^{-3_{x}} \quad x_{0}=2.5 \mu \mathrm{m} ;$ $C_{0}=16.9 \mathrm{nF} / \mathrm{cm}^{2}$ from $[4]$.

\section{Discussion and conclusion}

Comparison of the obtained expressions shows the strong influence of fractal medium (scaling factor $\alpha$ ) on the electric field value and charge density in the semiconductor that is followed by changes of capacitive device characteristics.

The differential-integral approach developed for MOS structures leads to fractional order equations describing the process of charge accumulation at the metal-oxide boundary.

The fractal distribution of charge carriers on the capacitance theoretically confirms the possibility of increasing the capacitance of MOS structures, in particular, to increase the efficiency of the FET.

An analysis of the obtained equations solutions shows that scaling allows us to control and regulate the degree of charge and capacity accumulation by a nanosheet in a natural and artificially created environment of a metamaterial, the geometric or physical properties of which are modeled by fractal physical charges (current) elements in ferroelectric and semiconducting metamaterials.

\section{References}

1. Khan, A.I. Negative capacitance in a ferroelectric capacitor /A.I.Khan et al. // Nat. Mater. - 2015. - Vol. 14. - P. 182 - 186.

2. Mengwei, S. Step-slope hysteresis-free negative capacitance MoS2 transistors / S. Mengwei et al. // Nature Nanotechnology. - 2018. -Vol. 13, P. 24 - 28.

3. Kobayashi, M. On device design for steep-slope negative capacitance field-effect transistor operating at sub- $0.2 \mathrm{~V}$ supply voltage with ferroelectric $\mathrm{HfO}_{2}$ thin film / M. Kobayashi and T. Hiramoto // AIP Advances. -2016. - Vol. 6, P. 025113.

4. Cobbold, R. Theory and Application of Field-effect Transistors / R.Cobbold. Wiley-Interscience, 1970.

5. Onufrienko, V.M. Potentials of fractal charges and currents in artificial medium / V.M. Onufrienko / RadioElectronics, Computer Science, Control. -2004. - Vol. 1.1, No. 1. - P. $18-21$.

6. Onufriyenko, V.M. Electromagnetism of artificial fractal medium - the physicogeometrical groundwork / V.M. Onufriyenko // Proc.6th Int. Symp. (Kharkov, Ukraine, June 2007). - P. 947 - 949.

7. Fikhtengolts, G.M. Kurs differentsialnogo I integralnogo ischisleniya. Tom 1 / G.M. Fikhtengolts. - Moscow: Nauka, 1962.

8. Ginzburg, A.I. Fractional integro-differentiation in Hölder classes of variable order / A.I. Ginzburg, N.K. Karapetianz // Doklady AN. -1994. - Vol. 339, No. 4. - P. $439-441$.

9. Onufrienko, V.M. Differintegral model of field-effect tranzistor with fractal nanolayer of channel / V.M. Onufrienko, L.M. Onufrienko // Proc. VII Intern. Scient.Pract. Conf. (Chernivtsi, Ukraine, November 2018). - P. 113 - 114.

10. Onufrienko, V.M. Modeling Characteristics of Field-Effect Fractal Nanotransistor / V.M. Onufrienko, T.I. Slyusarova, L.M. Onufrienko // Proc. 15th Int. Conf. on Advanced Trends in Radioelectronics, Telecom. and Comp. Engineering (Lviv - Slavske, Ukraine, February 2020). - P. 586 - 589. 